Arq. Bras. Med. Vet. Zootec., v.70, n.2, p.535-544, 2018

\title{
A simulação da biomassa de aveia por elementos climáticos, nitrogênio e regulador de crescimento
}

[The simulation of the oat biomass by climatic elements, nitrogen and growth regulator]

\author{
A. Marolli, J.A.G. da Silva, S. Sawicki, M.O. Binelo, A.H. Scremin, D.C. Reginatto, \\ E.F. Dornelles, D.M. Lambrecht
}

Universidade Regional do Noroeste do Estado do Rio Grande do Sul - Ijuí, RS

\section{RESUMO}

A produção de biomassa de aveia voltada à elaboração de silagem de qualidade é dependente de elementos climáticos e nitrogênio sem ocorrência de acamamento. O objetivo do presente estudo é a definição da dose ideal do regulador de crescimento que possibilite, no máximo, 5\% de acamamento de plantas de aveia, bem como a identificação das variáveis potenciais para composição do modelo de regressão linear múltipla com simulação da produtividade de biomassa à elaboração de silagem nas condições de uso do regulador, em reduzida, alta e muito alta fertilização com nitrogênio. O estudo foi conduzido em 2013, 2014 e 2015, em delineamento de blocos ao acaso, com quatro repetições em esquema fatorial $4 \times 3$, para doses de regulador $\left(0,200,400\right.$ e $\left.600 \mathrm{~mL} \mathrm{ha}^{-1}\right)$ e doses de nitrogênio $(30,90$ e $\left.150 \mathrm{~kg} \mathrm{ha}^{-1}\right)$, respectivamente. A dose de $495 \mathrm{~mL} \mathrm{ha}^{-1}$ de regulador se mostra eficiente na redução do acamamento de plantas de aveia em condição de reduzida, alta e muito alta fertilização com nitrogênio. A soma térmica, a precipitação, a radiação, a dose de regulador e o nitrogênio qualificam a composição do modelo de regressão linear múltipla, tornando eficiente a simulação da produtividade de biomassa da aveia para silagem ao longo do ciclo.

Palavras-chave: Avena sativa, ensilagem, trinexapac-ethyl, regressão linear múltipla

\begin{abstract}
The production of oat biomass focused on the development of quality silage is dependent on climatic elements and nitrogen without lodging occurrence. The objective of the study is to define the optimal dose of growth regulator that allows a maximum of 5\% oat plant lodging and identify potential variables for composition of multiple linear regression model with productivity simulation of biomass to the preparation of silage in the conditions of low, high and very high fertilization with nitrogen. The study was conducted in 2013, 2014, and 2015 in the randomized block design with four replications in a factorial $4 \times 3$, for regulator doses $\left(0,200,400\right.$ and $\left.600 \mathrm{ml} \mathrm{ha}^{-1}\right)$ and nitrogen doses $\left(30,90 \mathrm{and} 150 \mathrm{~kg} \mathrm{ha}^{-}\right.$

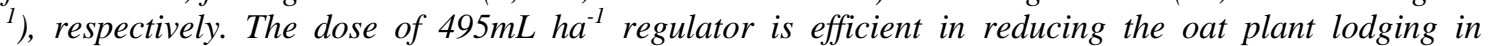
condition reduced, high, and very high fertilization with nitrogen. Thermal time, precipitation, radiation, regulator dose and nitrogen dose qualify the composition of the multiple linear regression model, making efficient the biomass oat productivity simulation for silage over the cycle.
\end{abstract}

Keywords: Avena sativa, silage, trinexapac-ethyl, linear multiple regression

\section{INTRODUÇ̃̃O}

A silagem é o processo de conservação da forragem resultante da fermentação anaeróbica, em que, basicamente carboidratos solúveis são convertidos em ácidos orgânicos pela ação de microrganismos que se proliferam e proporcionam condições adequadas à conservação, preservando o valor nutritivo (Zamarchi et al., 2014). Portanto, a ensilagem de gramíneas forrageiras é uma alternativa para fornecimento de volumoso de qualidade em períodos de baixa oferta de pasto no campo

Recebido em 21 de outubro de 2016

Aceito em 24 de maio de 2017

E-mail: marollia@yahoo.com.br 
(Paris et al., 2015). A aveia branca, além de ser excelente forragem para oferta de pastejo direto aos animais, é uma excelente alternativa de inverno para o processo de ensilagem (Zamarchi et al., 2014).

A alta produtividade de biomassa de aveia está associada ao desempenho das cultivares, às tecnologias de manejo, ao clima e ao solo favorável (Fontanelli et al., 2012; Silva et al., 2015). Entre as tecnologias de manejo, a adubação nitrogenada ganha destaque, pois estimula o crescimento de parte aérea e de raízes com reflexos expressivos sobre a produtividade (Costa et al., 2013; Mantai et al., 2015). No entanto, em anos desfavoráveis, a eficiência de uso do nutriente pode ser comprometida, o que reduz a produtividade e aumenta os custos de produção com poluição ambiental (Benin et al., 2012; Silva et al., 2015). Além disso, o incremento de nitrogênio para maior produtividade, junto às condições climáticas favoráveis, aumenta o crescimento vegetativo da planta, facilitando, assim, a ocorrência do acamamento (Flores et al., 2012; Silva et al., 2015).

O acamamento é um fenômeno complexo, no qual a planta perde sua posição vertical, inclinase e cai sobre o solo, afetando a produtividade e a qualidade das forragens, além de dificuldades na colheita (Silva et al., 2012; Hawerroth et al., 2015). A expressão do acamamento depende de fatores genéticos inter-relacionados com fatores externos, como vento, chuva, solo, densidade de plantas e técnicas de manejo (Silveira et al., 2011; Silva et al., 2015). Para minimizar a ocorrência do acamamento de plantas, tem-se avaliado o uso de reguladores de crescimento, como o trinexapac-ethyl, em culturas como arroz (Arf et al., 2012) e trigo (Schwerz et al., 2015). Reguladores de crescimento são compostos químicos que promovem a redução do comprimento do colmo pela obstrução da biossíntese de ácido giberélico, o que torna a planta mais adaptada e eficiente sobre os estímulos de produção (Kaspary et al., 2015; Hawerroth et al., 2015).

As relações entre os elementos climáticos e a produtividade de biomassa da aveia podem favorecer a construção de modelos de simulação da produtividade de forragem à elaboração de silagem, o que contribui para o manejo mais eficiente do nitrogênio com o uso do regulador de crescimento à alta produtividade de biomassa. Dentre os modelos de simulação, a regressão linear múltipla possibilita a combinação de diversos fatores para estimativa da produtividade (Godoy et al., 2015). Dalchiavon et al. (2012), mediante a utilização da regressão múltipla, estimaram a produtividade de arroz, incorporando no modelo o número de espiguetas da panícula. Leilah e Khateeb (2005) simularam a produtividade de trigo em condição de seca pela massa e pelo comprimento de grãos por espiga, índice de colheita, número de espigas por área e produção de biomassa. Portanto, o uso de modelos eficientes que integrem componentes da planta, do manejo e do clima pode contribuir para a previsibilidade de safras agrícolas e o planejamento de colheita, principalmente voltada à elaboração de biomassa para silagem.

O objetivo do presente estudo é a definição da dose ideal do regulador de crescimento que possibilite acamamento de plantas de aveia inferior a 5\%, bem como a identificação de elementos climáticos potenciais sobre 0 desenvolvimento da aveia para composição do modelo de regressão linear múltipla e a simulação da produtividade de biomassa à elaboração de silagem nas condições de uso do regulador de crescimento em reduzida, alta e muito alta fertilização com nitrogênio.

\section{MATERIAL E MÉTODOS}

O trabalho foi desenvolvido a campo, nos anos agrícolas de 2013, 2014 e 2015, em Augusto Pestana, RS, Brasil (28 26' 30' ' latitude S e $54^{\circ}$ 00' 58', longitude W). O solo da área experimental é classificado como Latossolo Vermelho Distroférrico típico, e o clima, segundo classificação de Köppen, do tipo Cfa, com verão quente sem estação seca. Aos 10 dias antes da semeadura, foi realizada análise de solo, que identificou, na média dos anos, as seguintes características químicas do local: $\mathrm{pH}=6,2$, $\mathrm{P}=33,9 \mathrm{mg} \mathrm{dm} \mathrm{dm}^{-3}, \mathrm{~K}=200 \mathrm{mg} \mathrm{dm} \mathrm{dm}^{-3}, \mathrm{MO}=3,0 \%$, $\mathrm{Al}=0 \quad \mathrm{cmol}_{\mathrm{c}} \quad \mathrm{dm}^{-3}, \quad \mathrm{Ca}=6,5 \mathrm{cmol}_{\mathrm{c}} \quad \mathrm{dm}^{-3} \quad \mathrm{e}$ $\mathrm{Mg}=2,5 \mathrm{cmol}_{\mathrm{c}} \mathrm{dm}^{-3}$. A semeadura foi realizada com semeadura-adubadora em sistema soja/aveia. Foram aplicados, na semeadura, $30 \mathrm{e}$ $20 \mathrm{~kg} \mathrm{ha}^{-1}$ de $\mathrm{P}_{2} \mathrm{O}_{5}$ e $\mathrm{K}_{2} \mathrm{O}$, respectivamente, com base nos teores de $\mathrm{P}$ e $\mathrm{K}$ no solo para expectativa de produtividade de grãos de $3 \mathrm{t} \mathrm{ha}^{-1}$ e de $\mathrm{N}$ na base com $10 \mathrm{~kg} \mathrm{ha}^{-1}$, sendo o restante para 
contemplar as doses de estudo aplicado no estádio de quarta folha expandida, com nitrogênio disponibilizado na forma de ureia. As sementes foram submetidas ao teste de germinação e vigor em laboratório, a fim de se corrigir a densidade de 400 sementes viáveis $\mathrm{m}^{-2}$. Durante a execução do estudo, foram efetuadas duas aplicações do fungicida tebuconazole, de nome comercial FOLICUR ${ }^{\circledR} \mathrm{CE}$, na dosagem de $0,75 \mathrm{~L} \mathrm{ha}^{-1}$. Além disso, o controle de plantas daninhas foi efetuado com herbicida metsulfuron-metil, de nome comercial ALLY ${ }^{\circledR}$, na dose de $4 \mathrm{~g} \mathrm{ha}^{-1}$, e capinas adicionais sempre que necessário. $\mathrm{O}$ regulador de crescimento (trinexapac-ethyl) foi aplicado com pulverizador costal à pressão constante de $30 \mathrm{lb} \mathrm{pol}^{-2}$, pelo $\mathrm{CO}_{2}$ comprimido, com pontas de jato plano "leque", no estádio do primeiro e do segundo nó visível do colmo.

O delineamento experimental foi o de blocos ao acaso, com quatro repetições, em esquema fatorial $4 \times 3$, nas fontes de variação doses de regulador de crescimento $(0,200,400$ e $600 \mathrm{~mL}$ $\left.\mathrm{ha}^{-1}\right)$ e doses de nitrogênio $\left(30,90\right.$ e $\left.150 \mathrm{~kg} \mathrm{ha}^{-1}\right)$, respectivamente, totalizando 48 unidades experimentais constituídas de cinco linhas, com $5 \mathrm{~m}$ de comprimento e espaçadas $0,20 \mathrm{~m}$, gerando a parcela de $5 \mathrm{~m}^{2}$. A colheita dos experimentos ocorreu de forma manual, pelo corte de $1 \mathrm{~m}^{2}$ de cada parcela a cada 30 dias. As plantas foram direcionadas à estufa de ar forçado à temperatura de $65^{\circ} \mathrm{C}$, até atingirem peso constante para pesagem e estimativa da produtividade biológica ( $\mathrm{PB}, \mathrm{kg} \mathrm{ha}^{-1}$ ). O acamamento (AC) foi estimado visualmente e expresso em porcentagem, considerado o ângulo formado na posição vertical do colmo das plantas em relação ao solo e à área de plantas acamadas, segundo metodologia sugerida por Moes e Stobbe (1991). As médias de produtividade de biomassa e grãos e as informações de temperatura e precipitação pluviométrica foram usadas na classificação dos anos em favorável e desfavorável ao cultivo.

Ao atender os pressupostos de homogeneidade e normalidade via testes de Bartlett, foi realizada análise de variância para detecção dos efeitos principais e de interação. Foi realizado ajuste da equação de regressão linear, que descreve o comportamento da expressão do acamamento de plantas de aveia pelo incremento das doses de regulador de crescimento. Nessas equações, foi considerada a possibilidade de acamamento de plantas de, no máximo, $5 \%$, valor adicionado ao parâmetro "y" da equação, obtida por $\mathrm{x}=[(\mathrm{y}-$ $\left.\mathrm{b}_{0}\right) /\left( \pm \mathrm{b}_{1}\right)$ ]. Procedeu-se à escolha das variáveis potenciais para o modelo de regressão linear múltipla via técnica de StepWise. Esse procedimento constrói iterativamente uma sequência de modelos de regressão pela adição e remoção de variáveis, selecionando aquelas que possuem maior relação com a variável principal (y), mediante a utilização da estatística de F parcial, conforme modelo:

$$
F_{j}=\frac{S Q_{R}\left(\beta_{j} \mid \beta_{1}, \beta_{0}\right)}{M Q_{E}\left(x_{j}, x_{1}\right)}
$$

em que: $S Q_{R}$ é a soma quadrática da regressão e $M Q_{E}\left(x_{j}, x_{1}\right)$ é a média quadrática do erro para o modelo contendo as variáveis $x_{1}$ e $x_{j}$. As variáveis selecionadas via StepWise foram utilizadas para se determinar a equação de regressão linear múltipla, para a simulação da produtividade biológica de aveia, dadas por uma equação do tipo:

$$
y=b_{0} \pm b_{1} x \pm b_{2} x_{2} \pm \cdots \pm b_{n} x_{n}
$$

descrita na forma matricial como:

$$
y=\left[\begin{array}{l}
Y_{1} \\
Y_{2} \\
M \\
Y_{n}
\end{array}\right] ; X=\left[\begin{array}{ccccc}
1 & X_{11} & X_{12} & \ldots & X_{p 1} \\
1 & X_{21} & X_{22} & \ldots & X_{p 2} \\
M & M & M & \ldots & M \\
1 & X_{1 n} & X_{2 n} & \ldots & X_{p n}
\end{array}\right] ; \beta=\left[\begin{array}{c}
\beta_{0} \\
\beta_{1} \\
M \\
\beta_{n}
\end{array}\right] ; e \varepsilon=\left[\begin{array}{c}
\varepsilon_{1} \\
\varepsilon_{2} \\
M \\
\varepsilon_{n}
\end{array}\right]
$$

A partir dessas matrizes, é obtido o valor dos coeficientes de regressão, sendo:

$$
\hat{\beta}=\left(X^{\prime} X\right)^{-1} X^{\prime} Y
$$

e a variância desses coeficientes é obtida pela matriz de covariância do vetor dos coeficientes de regressão:

$$
\operatorname{Cov} v(\hat{\beta})=\left(X^{\prime} X\right)^{-1} \hat{\sigma}^{2}
$$




$$
\hat{\sigma}^{2}=\frac{(Y-X \hat{\beta})(Y-X \hat{\beta})}{n-p-1}
$$

sendo $n$ o número de equações e $p$ o número de parâmetros. $\mathrm{O}$ teste de hipótese verificou $H_{0}: \beta_{i}=0$ vs $H_{a}: \beta_{i} \neq 0$, expresso por:

$$
t=\frac{\hat{\beta}_{i}-\beta_{i}}{\sqrt{\hat{V}\left(\hat{\beta}_{i}\right)}}
$$

\section{RESULTADOS E DISCUSSÃO}

Na Fig. 1, no momento de aplicação de nitrogênio em 2014, a média de temperatura máxima se mostrou a mais elevada em relação a 2015 e 2013. Além disso, a aplicação de nitrogênio foi seguida de precipitações pluviométricas superiores a $50 \mathrm{~mm}$, volume também observado próximo à colheita de grãos. Esses fatos justificam a baixa produtividade obtida (Tab. 1), caracterizando o ano de 2014 como desfavorável ao cultivo (AD).

Para essas análises, foi utilizado o programa computacional Genes (Cruz, 2013).
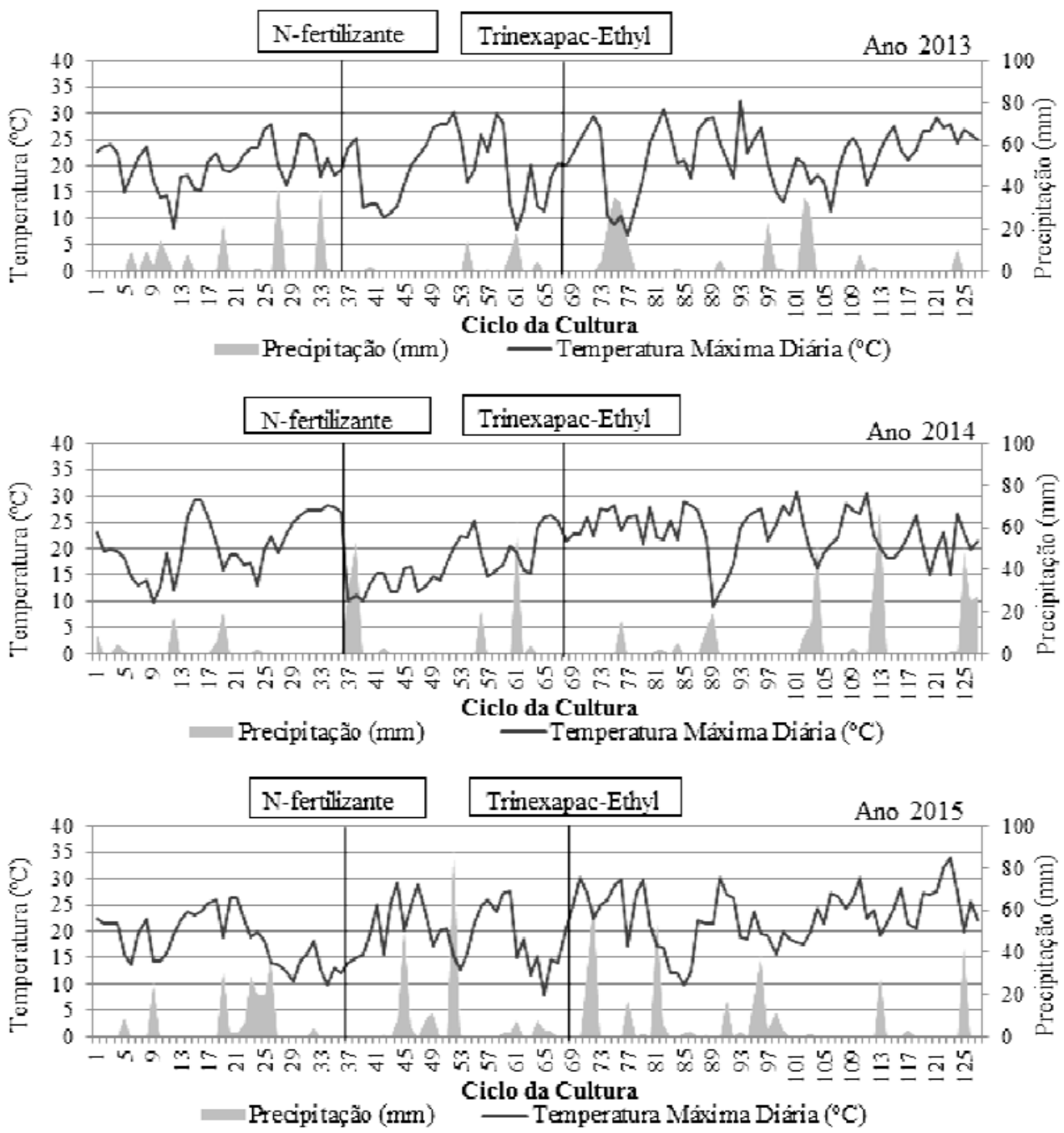

Figura 1. Precipitação pluviométrica, temperatura máxima no ciclo de aveia, aplicação de $\mathrm{N}$-fertilizante e aplicação de trinexapac-ethyl. 
O ano de 2015 evidenciou a menor temperatura máxima próxima à aplicação de nitrogênio. No momento da aplicação do nitrogênio, o solo se apresentava com condições de umidade favorável pelo acúmulo de chuvas dos dias anteriores (Fig. 1). O elevado volume de chuvas durante o ciclo proporcionou períodos de menor insolação, reduzindo a eficiência de fotossintese, o que, somado às médias de produtividade (Tab. 1), caracterizou o ano como intermediário (AI) ao cultivo. Em 2013, a temperatura máxima na aplicação de nitrogênio se mostrou ideal, ao redor de $20^{\circ} \mathrm{C}$. A aplicação ocorreu em condições favoráveis de umidade do solo (Fig. 1). Embora o volume total de chuvas tenha sido o mais reduzido (Tab. 1), a adequada distribuição de precipitação ao longo do ciclo (Fig. 1) foi decisiva para a maior produtividade, com valores superiores a 4t ha ${ }^{-1}$ de grãos (Tab. 1), caracterizando 2013 como favorável (AF) ao cultivo.

Tabela 1. Dados de temperatura e precipitação nos meses e anos de cultivo de aveia e média de produtividade de biomassa e grãos com classificação dos anos agrícolas

\begin{tabular}{|c|c|c|c|c|c|c|c|c|c|}
\hline \multirow{2}{*}{ Ano } & \multirow{2}{*}{ Mês } & \multicolumn{3}{|c|}{ Temperatura } & \multicolumn{2}{|c|}{ Precipitação } & \multirow{2}{*}{$\mathrm{PG}_{\overline{\mathrm{x}}}$} & \multirow{2}{*}{$\mathrm{PB}_{\overline{\mathrm{x}}}$} & \multirow{2}{*}{ Classe } \\
\hline & & Mínima & Máxima & Média & Média* & Ocorrida & & & \\
\hline \multirow{7}{*}{2015} & Maio & 10,5 & 22,7 & 16,6 & 149 & 100 & \multirow{7}{*}{$3404 b$} & \multirow{7}{*}{$8450 b$} & \multirow{7}{*}{ AI } \\
\hline & Junho & 07,9 & 18,4 & 13,1 & 162 & 191 & & & \\
\hline & Julho & 08,3 & 19,2 & 13,7 & 135 & 200 & & & \\
\hline & Agosto & 09,3 & 20,4 & 14,8 & 138 & 223 & & & \\
\hline & Setembro & 09,5 & 23,7 & 16,6 & 167 & 046 & & & \\
\hline & Outubro & 12,2 & 25,1 & 18,6 & 156 & 211 & & & \\
\hline & Total & - & - & - & 909 & 973 & & & \\
\hline \multirow{7}{*}{2014} & Maio & 11,1 & 24,5 & 17,8 & 149 & 020 & \multirow{7}{*}{$2841 \mathrm{c}$} & \multirow{7}{*}{$7695 \mathrm{c}$} & \multirow{7}{*}{$\mathrm{AD}$} \\
\hline & Junho & 09,3 & 19,7 & 14,5 & 162 & 059 & & & \\
\hline & Julho & 07,4 & 17,5 & 12,4 & 135 & 176 & & & \\
\hline & Agosto & 12,9 & 23,4 & 18,1 & 138 & 061 & & & \\
\hline & Setembro & 12,0 & 23,0 & 17,5 & 167 & 194 & & & \\
\hline & Outubro & 15,0 & 25,5 & 20,2 & 156 & 286 & & & \\
\hline & Total & - & - & - & 909 & 798 & & & \\
\hline \multirow{7}{*}{2013} & Maio & 10,0 & 22,6 & 16,3 & 149 & 108 & \multirow{7}{*}{$4163 a$} & \multirow{7}{*}{$9373 a$} & \multirow{7}{*}{$\mathrm{AF}$} \\
\hline & Junho & 08,9 & 20,0 & 14,5 & 162 & 086 & & & \\
\hline & Julho & 07,0 & 20,6 & 13,8 & 135 & 097 & & & \\
\hline & Agosto & 06,6 & 19,8 & 13,2 & 138 & 163 & & & \\
\hline & Setembro & 09,6 & 21,0 & 15,3 & 167 & 119 & & & \\
\hline & Outubro & 13,2 & 27,1 & 20,2 & 156 & 138 & & & \\
\hline & Total & - & - & - & 909 & 712 & & & \\
\hline
\end{tabular}

*= Média histórica de precipitação pluviométrica obtida nos meses de maio a outubro de 1990 a 2015; médias seguidas de mesma letra na coluna não diferem entre si na probabilidade de 5\% de erro pelo teste de Scott e Knott; $\mathrm{AF}=$ ano favorável; $\mathrm{AD}=$ ano desfavorável; $\mathrm{AI}=$ ano intermediário; temperatura $\left({ }^{\circ} \mathrm{C}\right)$; precipitação $(\mathrm{mm}) ;$ $\mathrm{PG}_{\overline{\mathrm{x}}}=$ produtividade de grãos $\left(\mathrm{kg} \mathrm{ha}^{-1}\right) ; \mathrm{PB}_{\overline{\mathrm{x}}}=$ produtividade de biomassa $\left(\mathrm{kg} \mathrm{ha}^{-1}\right)$.

Em milho, muito utilizado para silagem, Storck et al. (2014) descrevem que a condição do ano de cultivo é grande responsável pela alteração da produtividade. Mantai et al. (2015), em aveia, comentam sobre a forte influência do ano agrícola sobre a produtividade. Battisti et al. (2013), em trigo, e Arenhardt et al. (2015), em aveia, relatam que a condição de ano agrícola é predominantemente definida pela distribuição e pelo volume de chuvas. A precipitação pluviométrica é a variável meteorológica que mais afeta a produtividade, pela sua interação com a temperatura, a insolação e a radiação. A temperatura, a luz e a radiação solar também são elementos que influenciam na produtividade (Silva et al., 2015). Em aveia, o clima favorável é descrito como aquele de temperaturas mais amenas e de qualidade de radiação em favorecer o afilhamento e o enchimento de grãos, sem ocorrência de chuvas em grande quantidade e intensidade, porém em quantidade suficiente para favorecer suprimento adequado de umidade armazenada no solo (Mantai et al., 2015). 
A proposta de simulação da produtividade de biomassa de aveia por ano agrícola não contempla modelos eficientes, haja vista a forte variação existente em cada ano de cultivo (Tab.1 e Fig.1). Desse modo, foi considerado o efeito cumulativo da variabilidade entre ano favorável, intermediário e desfavorável para obtenção de coeficientes mais ajustados aos modelos de regressão linear na estimativa da dose ideal de regulador e de regressão linear múltipla para simulação da produtividade de biomassa de aveia. Portanto, na Tab. 2, está apresentado o somatório dos valores meteorológicos em cada momento de corte junto às médias obtidas de produtividade de biomassa. No momento de corte aos 30 e 60 dias após a emergência, não houve diferenças na produtividade de biomassa com o incremento das doses de regulador em cada condição de uso de $\mathrm{N}$-fertilizante (Tab. 2).
Esse fato era esperado, visto que a aplicação do regulador de crescimento ocorreu aos 65 dias após a emergência, com o surgimento do primeiro e do segundo nó visível do colmo principal, conforme recomendação em trigo. A resposta ao uso de regulador sobre a expressão de biomassa já se mostrou efetiva aos 90 dias após a emergência. Nesse momento, a produtividade de biomassa evidenciou redução significativa nos pontos de 400 e $600 \mathrm{~mL} \mathrm{ha}^{-1}$, que não diferiram entre si, independentemente da condição de fertilização com nitrogênio. $\mathrm{O}$ corte de biomassa com 120 dias após a emergência indicou a mais efetiva redução da produtividade de biomassa com $600 \mathrm{~mL} \mathrm{ha}^{-1}$, com 30 e $90 \mathrm{~kg} \mathrm{ha}^{-}$ ${ }^{1}$ de nitrogênio. Na condição mais elevada de $\mathrm{N}$ fertilizante, a produtividade de biomassa foi significativamente reduzida com 400 e $600 \mathrm{~mL}$ ha $^{-1}$.

Tabela 2. Valores obtidos das variáveis meteorológicas e de produtividade de biomassa em diferentes momentos de corte no uso de nitrogênio e regulador de crescimento

\begin{tabular}{|c|c|c|c|c|c|c|}
\hline \multirow{2}{*}{$\begin{array}{l}\text { Variáveis } \\
\text { selecionadas }\end{array}$} & \multirow{2}{*}{$\begin{array}{l}\text { Dose N } \\
\left(\mathrm{kg} \mathrm{ha}^{-1}\right)\end{array}$} & \multirow{2}{*}{$\begin{array}{l}\text { Dose R } \\
\left(\mathrm{mmL} \mathrm{ha}^{-1}\right)\end{array}$} & \multicolumn{4}{|c|}{ Momento de corte (DAE) } \\
\hline & & & 30 & 60 & 90 & 120 \\
\hline \multirow{4}{*}{$\begin{array}{l}\text { Soma térmica (graus dia) } \\
\text { Precipitação }\left(\mathrm{mm} \mathrm{m}^{-2}\right) \\
\text { Radiação }\left(\mathrm{V} \mathrm{m}^{-1}\right)\end{array}$} & \multicolumn{6}{|c|}{$(2013+2014+2015)$} \\
\hline & - & - & 496 & 944 & 1452 & 1982 \\
\hline & - & - & 167 & 307 & 433 & 620 \\
\hline & - & - & 212 & 486 & 814 & 1160 \\
\hline \multirow{12}{*}{$\begin{array}{l}\text { Produtividade de biomassa } \\
\left(\mathrm{kg} \mathrm{h}^{-1}\right)\end{array}$} & \multirow{3}{*}{30} & 0 & $310 \mathrm{a}$ & $1813 \mathrm{a}$ & $8997 \mathrm{a}$ & $9629 \mathrm{a}$ \\
\hline & & 200 & $306 \mathrm{a}$ & $1849 \mathrm{a}$ & $8775 \mathrm{a}$ & $8562 \mathrm{~b}$ \\
\hline & & 400 & $295 \mathrm{a}$ & $1804 \mathrm{a}$ & $8022 \mathrm{~b}$ & $8421 \mathrm{~b}$ \\
\hline & \multirow{5}{*}{90} & 600 & $300 \mathrm{a}$ & $1816 \mathrm{a}$ & $8023 \mathrm{~b}$ & $7713 \mathrm{c}$ \\
\hline & & 0 & $296 \mathrm{a}$ & $1792 \mathrm{a}$ & $9370 \mathrm{a}$ & $11009 \mathrm{a}$ \\
\hline & & 200 & $282 \mathrm{a}$ & $1849 \mathrm{a}$ & $9030 \mathrm{a}$ & $10830 \mathrm{a}$ \\
\hline & & 400 & $272 \mathrm{a}$ & $1763 \mathrm{a}$ & $8155 \mathrm{~b}$ & $10147 \mathrm{~b}$ \\
\hline & & 600 & $262 \mathrm{a}$ & $1714 \mathrm{a}$ & $7909 \mathrm{~b}$ & $9027 \mathrm{c}$ \\
\hline & \multirow{4}{*}{150} & 0 & $295 \mathrm{a}$ & $1922 \mathrm{a}$ & $9157 \mathrm{a}$ & $11211 \mathrm{a}$ \\
\hline & & 200 & $292 \mathrm{a}$ & $1851 \mathrm{a}$ & 8726 a & $10988 \mathrm{a}$ \\
\hline & & 400 & $294 \mathrm{a}$ & $1887 \mathrm{a}$ & $7579 \mathrm{~b}$ & $10232 \mathrm{~b}$ \\
\hline & & 600 & $295 \mathrm{a}$ & $1870 \mathrm{a}$ & $7438 \mathrm{~b}$ & $9886 \mathrm{~b}$ \\
\hline
\end{tabular}

DAE: dias após a emergência. Dose R: doses de regulador de crescimento aplicado; dose N: doses de nitrogênio aplicado em cobertura; médias seguidas de mesma letra na coluna não diferem estatisticamente entre si em $5 \%$ de probabilidade de erro pelo teste de Scott e Knott.

$\mathrm{Na}$ Tab. 3, da estimativa da dose ideal de regulador de crescimento pela expressão do acamamento, as equações de regressão testadas apresentaram tendência linear decrescente significativa, independentemente da dose de nitrogênio. $\mathrm{Na}$ estimativa da dose ideal de regulador de crescimento, foi levada em consideração a possibilidade de acamamento de plantas de, no máximo, $5 \%$, valor adicionado ao parâmetro "y" de cada equação. Independentemente da condição de ano de cultivo, as doses de regulador de crescimento variaram em 460, 500 e $520 \mathrm{~mL} \mathrm{ha}^{-1}$ em condição de reduzida, alta e muito alta fertilização com nitrogênio, respectivamente. De modo geral, a dose de $495 \mathrm{ml} \mathrm{ha}^{-1}$ de regulador se mostrou efetiva na redução do acamamento, independentemente de ano agrícola e dose de nitrogênio. Em trigo, Pagliosa et al. (2013) e Trevizan et al. (2015), e em arroz, Arf et al. (2012) e Alvarez et al. (2014) observaram redução efetiva do acamamento de plantas com a dose de $400 \mathrm{~mL} \mathrm{ha}^{-1}$ de regulador. Em crotalária (Kappes et al., 2011) e em soja (Souza et al., 
2013), redução eficiente do acamamento foi obtida com $500 \mathrm{~mL} \mathrm{ha}^{-1}$ do regulador. Kaspary et al. (2015) e Guerreiro e Oliveira (2012), ao estudarem os efeitos do regulador de crescimento na produtividade de grãos e na qualidade de sementes de aveia, afirmam que a dose de $500 \mathrm{~mL} \mathrm{ha}^{-1}$ reduz a estatura da planta em até $60 \%$.

Tabela 3. Estimativa da dose ideal do regulador de crescimento por ano e dose de N-fertilizante na previsibilidade de no máximo $5 \%$ de acamamento

\begin{tabular}{|c|c|c|c|c|c|c|}
\hline $\begin{array}{l}\text { Dose N } \\
\left(\mathrm{kg} \mathrm{ha}^{-1}\right)\end{array}$ & Ano & $\begin{array}{c}\text { Equação } \\
\mathrm{AC}=\mathrm{a} \pm \mathrm{bx}\end{array}$ & $\mathrm{R}^{2}$ & $\mathrm{P}_{(\mathrm{bi})}$ & $\begin{array}{l}Y_{E} \\
(\%)\end{array}$ & $\begin{array}{l}\text { Dose ideal } \\
\left(\mathrm{mL} \mathrm{ha}^{-1}\right)\end{array}$ \\
\hline \multirow{3}{*}{30} & $2015(\mathrm{AI})$ & $23,55-0,045 x$ & 0,80 & $*$ & \multirow{4}{*}{ (5) } & $\cong 410$ \\
\hline & 2014 (AD) & $29,62-0,050 x$ & 0,92 & $*$ & & $\cong 495$ \\
\hline & 2013 (AF) & $22,52-0,037 x$ & 0,89 & $*$ & & $\cong 475$ \\
\hline$\overline{\mathrm{x}}_{30}$ & $2013+2014+2015$ & $25,23-0,044 x$ & 0,87 & $*$ & & $\cong 460$ \\
\hline \multirow{3}{*}{90} & $2015(\mathrm{AI})$ & $56,82-0,103 x$ & 0,91 & $*$ & \multirow{4}{*}{$(5)$} & $\cong 500$ \\
\hline & 2014 (AD) & $46,00-0,080 x$ & 0,82 & $*$ & & $\cong 510$ \\
\hline & 2013 (AF) & $48,75-0,088 x$ & 0,93 & $*$ & & $\cong 490$ \\
\hline $\bar{x}_{90}$ & $2013+2014+2015$ & $50,52-0,090 x$ & 0,88 & $*$ & & $\cong 500$ \\
\hline \multirow{3}{*}{150} & $2015(\mathrm{AI})$ & $82,35-0,147 x$ & 0,93 & $*$ & \multirow{4}{*}{$(5)$} & $\cong 525$ \\
\hline & 2014 (AD) & $71,25-0,127 x$ & 0,89 & $*$ & & $\cong 520$ \\
\hline & $2013(\mathrm{AF})$ & $75,15-0,133 x$ & 0,94 & $*$ & & $\cong 525$ \\
\hline$\overline{\mathrm{x}}_{150}$ & $2013+2014+2015$ & $76,25-0,135 x$ & 0,92 & $*$ & & $\cong 520$ \\
\hline$\overline{\mathrm{x}}_{\text {geral }}$ & $2013+2014+2015$ & $50,66-0,089 x$ & 0,89 & $*$ & $(5)$ & $\cong 495$ \\
\hline
\end{tabular}

*= Significativo a $5 \%$ de probabilidade de erro, respectivamente, pela probabilidade de $\mathrm{F} ; \mathrm{P}_{(\mathrm{bi})}=$ parâmetro que mede a inclinação da reta; $\mathrm{R}^{2}=$ coeficiente de determinação; ( ) = consideração da possibilidade de acamamento de plantas inferior a $5 \% ; \overline{\mathrm{x}}_{\mathrm{N}}=$ média obtida nos três anos de estudo; $\overline{\mathrm{x}}_{\text {geral }}=$ média geral; $\mathrm{Y}_{\mathrm{E}}=$ valor estimado; dose ideal= dose de regulador que possibilita acamamento de plantas inferior a $5 \%$.

$\mathrm{Na}$ Tab. 4, as variáveis potenciais para composição do modelo de regressão linear múltipla foram a soma térmica, a precipitação pluviométrica, a radiação térmica e o momento de corte, em todas as condições de uso de regulador crescimento e de $\mathrm{N}$-fertilizante. $\mathrm{Na}$ proposta de um modelo mais amplo, com a possibilidade de simulação com definição de uma dose de regulador no intervalo de 0 a $600 \mathrm{~mL} \mathrm{ha}^{-1}$, o efeito do produto trinexapac-ethyl foi significativo para compor o modelo múltiplo, independentemente da dose de $\mathrm{N}$-fertilizante. $\mathrm{Na}$ validação de um modelo mais completo, envolvendo o uso combinado do nitrogênio e do regulador, as variáveis: dose de regulador de crescimento e nitrogênio foram significativas para compor o modelo múltiplo junto com os elementos meteorológicos e a biomassa produzida ao longo do ciclo de desenvolvimento da aveia. A identificação de componentes que influenciam na produtividade é decisiva na elaboração de modelos de simulação (Leal et al., 2015). O uso da técnica StepWise pode qualificar a seleção de variáveis potenciais na simulação por regressão linear múltipla (Balbinot et al., 2005). Modelos meteorológicos permitem estimativas da produtividade anteriores à colheita, constituindo, assim, uma importante ferramenta na previsão de safras agrícolas (Melo et al., 2008). Para Silva Junior et al. (2012), os fatores temperatura, radiação e precipitação são os principais agentes reguladores da produção agrícola. 
Tabela 4. Identificação de variáveis potenciais via StepWise para composição do modelo de regressão linear múltipla à simulação da produtividade de biomassa de aveia

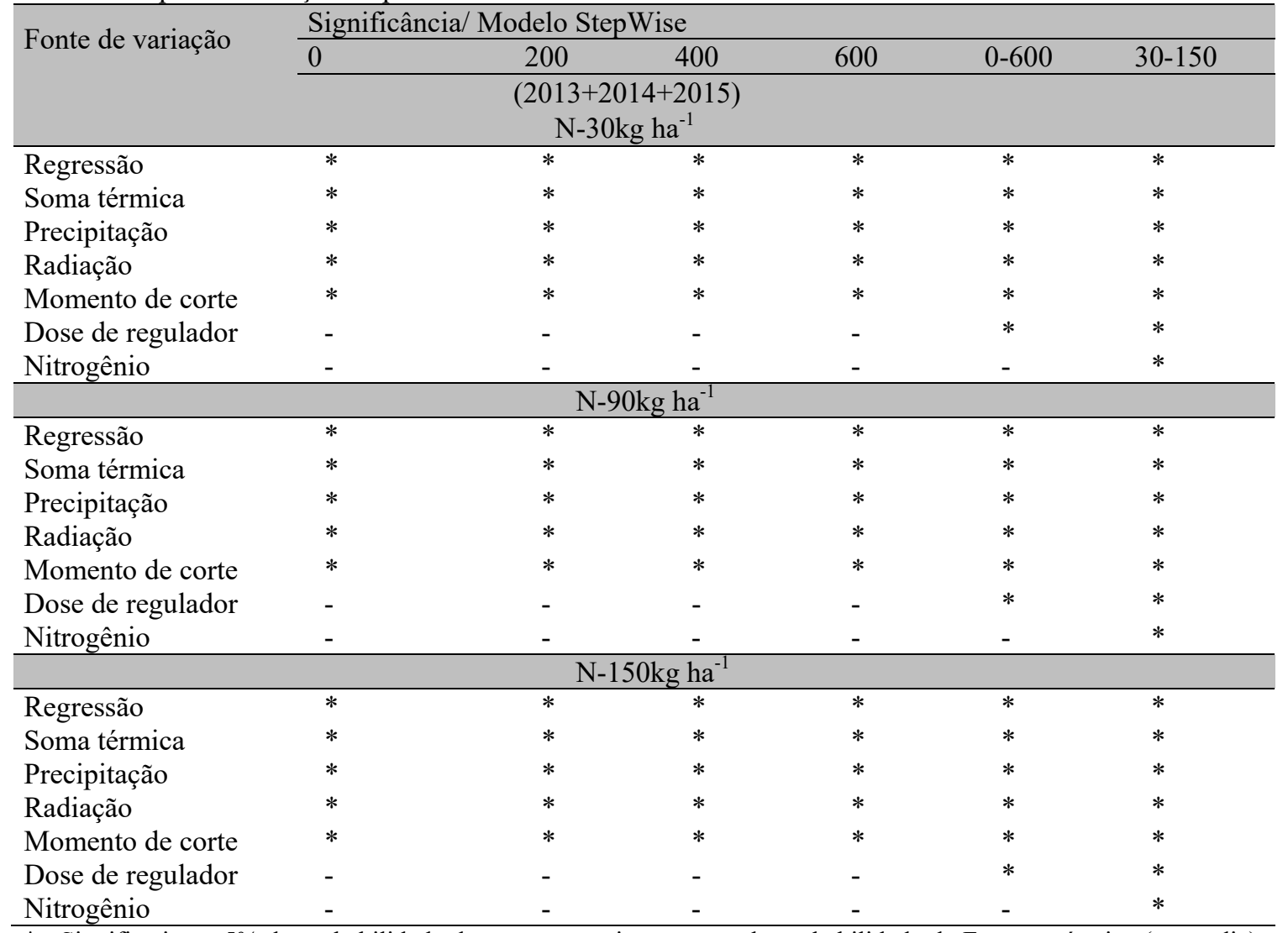

*= Significativo a 5\% de probabilidade de erro, respectivamente, pela probabilidade de F; soma térmica (graus dia); precipitação $\left(\mathrm{mm} \mathrm{m}^{-2}\right)$; radiação $\left(\mathrm{V} \mathrm{m}^{-1}\right)$; momento de corte (dias); Dose de regulador: dose ideal de regulador para estimativa de acamamento inferior a $5 \%\left(\mathrm{~mL} \mathrm{ha}^{-1}\right)$; nitrogênio $\left(\mathrm{kg} \mathrm{ha}^{-1}\right)$.

$\mathrm{Na}$ Tab. 5, estão apresentadas as equações de regressão linear múltipla para simulação da produtividade de biomassa de aveia. Nessa simulação, foram empregados os valores apresentados na Tab. 2 junto à dose ideal de regulador de crescimento da Tab. 3. Na dose de $30 \mathrm{~kg} \mathrm{ha}^{-1}$ de nitrogênio, o incremento da dose de regulador de crescimento proporciona diminuição da produtividade de biomassa, com valores estimados variando entre 9625 e $7728 \mathrm{~kg}$ $\mathrm{ha}^{-1}$, inclusive, as equações testadas se mostraram altamente eficientes, com valores muito próximos aos observados em campo. $\mathrm{Na}$ análise do modelo mais amplo, com inclusão da dose ideal de regulador de crescimento (Tab. 3), o valor estimado de produtividade de biomassa é muito próximo ao observado e dentro do intervalo de confiança proposto. $\mathrm{Na}$ análise do modelo mais completo que inclui os elementos climáticos, o manejo pelo nitrogênio e o regulador de crescimento, a eficiência na simulação da produtividade de biomassa foi confirmada, validando uma proposta de qualificar a previsibilidade de safra sobre a aveia direcionada à elaboração de silagem. 
Tabela 5. Regressão linear múltipla para estimativa da produtividade de biomassa por elementos climáticos, doses de nitrogênio e regulador de crescimento no ciclo da aveia

\begin{tabular}{|c|c|c|c|c|c|}
\hline \multirow{2}{*}{$\begin{array}{l}\text { Dose R } \\
\left(\mathrm{mL} \mathrm{ha} \mathrm{a}^{-1}\right)\end{array}$} & \multirow{3}{*}{$\begin{array}{c}\text { Equação } \\
\mathrm{PB}=\mathrm{b}_{0} \pm \mathrm{b}_{1} \mathrm{x} \pm \mathrm{b}_{2} x_{2} \pm \cdots \pm \mathrm{b}_{\mathrm{n}} x_{n}\end{array}$} & \multicolumn{2}{|l|}{ PB } & \multicolumn{2}{|l|}{ IC } \\
\hline & & \multirow[t]{2}{*}{$\mathrm{E}$} & \multirow[t]{2}{*}{$\mathrm{O}$} & \multirow[t]{2}{*}{ LI } & \multirow[t]{2}{*}{ LS } \\
\hline \multirow{2}{*}{\multicolumn{6}{|c|}{$\frac{(2013+2014+2015)}{\mathrm{N}-30 \mathrm{~kg} \mathrm{ha}^{-1}}$}} \\
\hline & & & & & \\
\hline 0 & $\mathrm{~PB}=-271+26,4_{\mathrm{Ra}}-0,9_{\mathrm{Pr}}-10,8_{\mathrm{St}}+10,3_{\mathrm{Mc}}$ & 9625 & 9629 & 8613 & 10281 \\
\hline 200 & $\mathrm{~PB}=-209+23,7_{\mathrm{Ra}}-5,4_{\mathrm{Pr}}-16,3_{\mathrm{St}}+141,2_{\mathrm{Mc}}$ & 8572 & 8562 & 7439 & 10085 \\
\hline 400 & $\mathrm{~PB}=-188+22,3_{\mathrm{Ra}}-4,7_{\mathrm{Pr}}-14,6_{\mathrm{St}}+121,8_{\mathrm{Mc}}$ & 8445 & 8421 & 7314 & 9150 \\
\hline 600 & $\mathrm{~PB}=-236+19,0_{\mathrm{Ra}}-4,2_{\mathrm{Pr}}-14,7_{\mathrm{St}}+147,2_{\mathrm{Mc}}$ & 7728 & 7713 & 7155 & 8358 \\
\hline $0-600$ & $\mathrm{~PB}=61-0,9_{\mathrm{DR}}+22,4_{\mathrm{Ra}}-4,7_{\mathrm{Pr}}-14,2_{\mathrm{St}}+117_{\mathrm{Mc}}$ & 8612 & 8581 & 8160 & 9080 \\
\hline \multicolumn{6}{|c|}{ N-90kg ha ${ }^{-1}$} \\
\hline 0 & $\mathrm{~PB}=-211+29,8_{\mathrm{Ra}}-6,3_{\mathrm{Pr}}-13,6_{\mathrm{St}}+62,5_{\mathrm{Mc}}$ & 10208 & 11009 & 9574 & 11427 \\
\hline 200 & $\mathrm{~PB}=-189+29,9_{\mathrm{Ra}}-6,5_{\mathrm{Pr}}-12,4_{\mathrm{St}}+38,3_{\mathrm{Mc}}$ & 9827 & 10530 & 9280 & 11130 \\
\hline 400 & $\mathrm{~PB}=-232+29,5_{\mathrm{Ra}}-5,8_{\mathrm{Pr}}-13,8_{\mathrm{St}}+58,5_{\mathrm{Mc}}$ & 9665 & 10147 & 8820 & 10510 \\
\hline 600 & $\mathrm{~PB}=-279+22,2_{\mathrm{Ra}}-4,2_{\mathrm{Pr}}-6,7_{\mathrm{St}}-4,6_{\mathrm{Mc}}$ & 8982 & 9027 & 8061 & 9788 \\
\hline $0-600$ & $\mathrm{~PB}=-229+0,01_{\mathrm{DR}}+27,9_{\mathrm{Ra}}-5,7_{\mathrm{Pr}}-11,6_{\mathrm{St}}+38_{\mathrm{Mc}}$ & 10171 & 10178 & 9681 & 10670 \\
\hline \multicolumn{6}{|c|}{$\mathrm{N}-150 \mathrm{~kg} \mathrm{ha}^{-1}$} \\
\hline 0 & $\mathrm{~PB}=-205+27,9_{\mathrm{Ra}}-6,1_{\mathrm{Pr}}-16,7_{\mathrm{St}}+132_{\mathrm{Mc}}$ & 11118 & 11211 & 9495 & 11965 \\
\hline 200 & $\mathrm{~PB}=-164+31,7_{\mathrm{Ra}}-6,4_{\mathrm{Pr}}-12,7_{\mathrm{St}}+27,2_{\mathrm{Mc}}$ & 10733 & 10908 & 9358 & 11886 \\
\hline 400 & $\mathrm{~PB}=-133+29,4_{\mathrm{Ra}}-6,4_{\mathrm{Pr}}-17,5_{\mathrm{St}}+127_{\mathrm{Mc}}$ & 10558 & 10532 & 8630 & 11363 \\
\hline 600 & $\mathrm{~PB}=-101+34,6_{\mathrm{Ra}}-6,5_{\mathrm{Pr}}-12,3_{\mathrm{St}}-11,3_{\mathrm{Mc}}$ & 10270 & 10386 & 8217 & 11283 \\
\hline $0-600$ & $\mathrm{~PB}=-155+0,01_{\mathrm{DR}}+30,9_{\mathrm{Ra}}-6,2_{\mathrm{Pr}}-14_{\mathrm{St}}+55_{\mathrm{Mc}}$ & 10702 & 10760 & 9720 & 11016 \\
\hline Geral & $\mathrm{PB}=-182+0,83_{\mathrm{N}}-0,32_{\mathrm{DR}}+27_{\mathrm{Ra}}-5,65_{\mathrm{Pr}}-13,5_{\mathrm{St}}+71,4_{\mathrm{Mc}}$ & 9346 & 9640 & 9300 & 9964 \\
\hline
\end{tabular}

$\mathrm{PB}=$ produtividade de biomassa $\left(\mathrm{kg} \mathrm{ha}^{-1}\right) ; \mathrm{ST}=$ soma térmica (graus dia); $\mathrm{Pr}=$ precipitação $\left(\mathrm{mm} \mathrm{m}^{2}\right) ; \mathrm{RA}=$ radiação $\left(\mathrm{V} \mathrm{m}^{-1}\right) ; \mathrm{MC}=$ momento de corte (dias); Geral = modelo geral, independente da condição de adubação nitrogenada $\mathrm{e}$ dose de regulador de crescimento; $\mathrm{N}=$ nitrogênio $\left(70 \mathrm{~kg} \mathrm{ha}^{-1}\right) ; \mathrm{DR}=$ dose de regulador

\section{CONCLUSÕES}

A dose de $495 \mathrm{~mL} \mathrm{ha}^{-1}$ de regulador se mostra eficiente na redução do acamamento de plantas de aveia em condição de reduzida, alta e muito alta fertilização com nitrogênio. A soma térmica, a precipitação, a radiação, a dose de regulador e o nitrogênio qualificam a composição do modelo de regressão linear múltipla, tornando eficiente a simulação da produtividade de biomassa da aveia para silagem ao longo do ciclo.

\section{REFERÊNCIAS}

ALVAREZ, R.C.F.; CRUSCIOL, C.A.C.; NASCENTE, A.S. Produtividade de arroz de terras altas em função de reguladores de crescimento. Rev. Ceres, v.61, p.42-49, 2014.
ARENHARDT, E.G.; SILVA, J.A.G.; GEWEHR, E. et al. The nitrogen supply in wheat cultivation dependent on weather conditions and succession system in southern Brazil. Afr. J. Agric. Res., v.10, p.4322-4330, 2015.

ARF, O.; NASCIMENTO, V.; RODRIGUES, R.A.F. et al. Uso de etil-trinexapac em cultivares de arroz de terras altas. Pesqui. Agropecu. Trop., v.42, p.150-158, 2012.

BALBINOT, J.R.A.A.; BACKES, R.L.; ALVES, A.C. et al. Contribuição de componentes de rendimento na produtividade de grãos em variedades de polinização aberta de milho. Rev. Bras. Agrociênc., v.11, p.161$166,2005$.

BATTISTI, R.; SENTELHAS, P.C.; PILAU, F.G. et al. Eficiência climática para as culturas da soja e do trigo no estado do Rio Grande do Sul em diferentes datas de semeadura. Cienc. Rural, v.43, p.390-396, 2013. 
BENIN, G.; BORNHOFEN, E.; BECHE, E. et al. Agronomic performance of wheat cultivars in response to nitrogen fertilization levels. Acta Sci. Agron., v.34, p.275-283. 2012.

COSTA, L.; ZUCARELI, C.; RIEDE, C.R. Parcelamento da adubação nitrogenada no desempenho produtivo de genótipos de trigo. Rev. Cienc. Agron., v.44, p.215-224, 2013.

DALCHIAVON, F.C.; CARVALHO, M.P.E.; COLETTI, A.J. et al. Correlação linear entre componentes da produção e produtividade do arroz de terras altas em sistema de plantio direto. Cienc. Agrar., v.33, p.1629-1642, 2012.

FLORES, R.A.; URQUIAGA, S.S.; ALVES, B.J.R. et al. Adubação nitrogenada e idade de corte na produção de matéria seca do capim-elefante no Cerrado. Rev. Bras. Eng. Agric. Ambien., v.16, p.1282-1288, 2012.

FONTANELI, R.S.; SANTOS, H.P.; FONTANELI, R.S. et al. Rendimento de grãos de aveia branca em sistemas de produção com integração lavourapecuária, sob plantio direto. Rev. Bras. Cienc. Agrar., v.7, Supl., p.790-796, 2012.

GODOY, S. G. DE; STONE, L. F.; FERREIRA, E. P. DE B. et al. Correlação entre produtividade do arroz no sistema semeadura direta e atributos do solo. Rev. Bras. Eng. Agric. Ambien. v.19, p.119-125. 2015.

GUERREIRO M.G.; OLIVEIRA N.C. Produtividade de grãos de aveia branca submetida a doses de trinexapac-ethyl. Rev. Cienc. Exatas Terra Cienc. Agrar., v.7, p.27-36, 2012.

HAWERROTH, M.C.; SILVA, J.A.G.; SOUZA, C.A. et al. Redução do acamamento em aveia-branca com uso do regulador de crescimento etil-trinexapac. Pesqui. Agropecu. Bras., v.50, p.115-125, 2015.

KAPPES C.; ARF, O.; ARF, M.V. et al. Uso de reguladores de crescimento no desenvolvimento e produção de crotalária. Pesqui. Agropecu. Trop., v.41, p.508-518, 2011.

KASPARY, T.E.; LAMEGO, F.P.; BELLÉ, C. et al. Regulador de crescimento na produtividade $\mathrm{e}$ qualidade de sementes de aveia-branca. Planta Daninha, v.33, p.739-750, 2015.

LEAL, A.J.F.; MIGUEL, E.P.; BAIO, G.H.R. et al. Redes neurais artificiais na predição da produtividade de milho e definição de sítios de manejo diferenciado por meio de atributos do solo. Bragantia, v.74, p.436444, 2015.

LEILAH, A.A.; AL-KHATEEB, S.A. Statistical analysis of wheat yield under drought conditions. $J$. Arid Environ., v.61, p.483-496, 2005.
MANTAI, R.D.; SILVA, J.A.G.; ARENHARDT, E.G. et al. The effect of nitrogen dose on the yield indicators of oats. Afr. J. Agric. Res., v.10, p.3773$3781,2015$.

MELO, R.W.; FONTANA, D.C.; BERLATO, M.A. et al. An agrometeorological spectral model to estimate soybean yield, applied to southern Brazil. Int. J. Remote Sens, v.29, p.4013-4028, 2008.

MOES, J.; STOBBE, E.H. Barley treated with ethephon: I. yield components and net grain yield. Agron. J., v.83, p.86-90, 1991.

PAGLIOSA, E.E.; BENIN, G.; BIEZUS, E. et al. Trinexapac-Ethyl e adubação nitrogenada na cultura do trigo. Planta Daninha, v.31, p.623-630, 2013.

SCHWERZ, F.; CARON, B.O.; SCHMIDT, D. et al. Growth retardant and nitrogen levels in wheat agronomic characteristics. Científica, v.43, p.93-100, 2015.

SILVA JÚNIOR, E.B.; FERNANDES JÚNIOR, P.I.; OLIVEIRA, P.J. et al. Agronomic efficiency of a new rhizobial inoculant formulation for use in cowpea. Pesqui. Agropecu. Bras., v.47, p.138-141, 2012.

SILVA, J.A.G.; ARENHARDT, E.G.; KRÜGER, C.A.M.B. et al. A expressão dos componentes de produtividade do trigo pela classe tecnológica e aproveitamento do nitrogênio. Rev. Bras. Eng. Agric. Ambien., v.19, p.27-33, 2015.

SILVA, J.A.G.; FONTANIVA, C.; COSTA, J.P.S. et al. Uma proposta na densidade de semeadura de um biotipo atual de cultivares de aveia. Rev. Bras. Agrocienc., v.18, p.253-263, 2012.

SILVEIRA, C.P.; OLIVEIRA, D.A.B.; SILVA, E.M. et al. Two years of nitrogen and sulfur fertilizations in a signal grass pasture under degradation: changes in the root system. Rev. Bras. Zootec., v.40, p.11951203, 2011.

SOUZA, C.A.; FIGUEIREDO, B.P.; COELHO, C.M.M. et al. Plant architecture and productivity of soybean affected by plant growth retardants. Biosci. J., v.29, p.634-643, 2013.

STORCK L.; FILHO, A.C.; GUADAGNIN, J.P. Análise conjunta de ensaios de cultivares de milho por classes de interação genótipo $\mathrm{x}$ ambiente. Pesqui. Agropec. Bras., v.49, p.163-172, 2014.

TREVIZAN, K.; GREGOLETI, E.; HOFFMANN, A.F. Trinexapac-etil diminui o acamamento e aumenta produtividade do cultivar de trigo TBIO Pioneiro. Rev. Agron. Med. Vet., v.2, p.1-11, 2015.

ZAMARCHI, G.; PAULO S.P.; MENEZES, L.F.G. et al. Silage of white oat under nitrogen fertilization and pre-wilting. Cienc. Agrar., v.35, p.2185-2196, 2014. 